\title{
A novel GSH responsive poly(alpha-lipoic acid) nanocarrier bonding with the honokiol-DMXAA conjugate for combination therapy
}

\author{
Zhilin Liu ${ }^{1,2}$, Zhaohui Tang ${ }^{1 *}$, Dawei Zhang ${ }^{1}$, Jiatan $\mathrm{Wu}^{3}$, Xinghui $\mathrm{Si}^{1,2}, \mathrm{Na}$ Shen $^{1}$ and Xuesi Chen ${ }^{{ }^{*}}$
}

\begin{abstract}
The key to improve the therapeutic efficacy for cancer treatment is to increase the delivery of drugs to tumors. For this purpose, tumor-microenvironment stimuliresponsive materials have great potential. Here, we prepared a new nanomedicine by bonding the conjugate of honokiol (HNK) and 5,6-dimethylxanthenone-4-acetic acid (DMXAA) to a glutathione (GSH)-responsive nanocarrier, poly $(\alpha$-lipoic acid) polyethylene glycol. The nanomedicine would disintegrate due to the high level of GSH at the tumor sites, achieving the co-delivery of HNK and DMXAA, and realizing the combination therapy through close-range killing by HNK and long-range striking by DMXAA together. In a murine 4T1 breast tumor model, this strategy exhibited high tumor inhibition rate of $93 \%$, and provided a valuable therapeutic choice for cancer therapy.
\end{abstract}

Keywords: glutathione response, conjugate, co-delivery and antitumor

\section{INTRODUCTION}

The combination therapy in cancer treatment achieved rapid development in both research and clinical practice $[1,2]$. As we all know, due to the poor permeability of cytotoxic drugs in solid tumors, more and more attention has been paid to the combination therapy consisting of vascular disrupting agents (VDAs) [3,4], which can block the nutrients and oxygen supply by destroying the tumor vessels. As one of the most famous leading VDAs, 5,6dimethylxanthenone-4-acetic acid (DMXAA) $[5,6]$ can induce hemorrhagic necrosis due to the ability to inhibit blood flow selectively at the tumor sites, which can increase vascular endothelial apoptosis, induce coordinated decreases in tumor blood flow and enhance vascular permeability of tumors. As VDAs can cause extensive and concentrated necrosis of the tumors and eventually achieve the long-range strike of the tumors, which just makes up for the shortcomings of cytotoxic drugs that cannot penetrate into the tumor center sufficiently, the combination strategy of VDAs and cytotoxic drugs in the treatment of cancer has achieved encouraging results $[7,8]$.

Tumor-microenvironment responsive nanocarriers have shown great potentials to increase the delivery of drugs to tumors [9-11]. Considering that both cytotoxic drugs and VDAs need to reach the tumor site to produce a marked effect, we can use the tumor-microenvironment-responsive nanocarriers to enhance the selective release of cytotoxic drugs and VDAs at the tumor sites. According to the characteristics of tumor microenvironment, such as enzymes [12] and $\mathrm{pH}$ [13-15] differences, high reactive oxygen species (ROS) [16-18] and L-glutathione (GSH) [19] level, various stimuli-responsive nanocarriers have been designed. Of these aspects, as the GSH concentration of the malignant tissues is at least four-fold higher than that of the normal tissues [20-22], and the intracellular concentration of GSH is much higher than that in most extracellular fluids [23,24], many new GSH responsive nanoparticles achieved selective release of drugs at tumors [25]. However, most of them were prepared by complex process, such as modifying side groups [26]. In our recent study, GSH responsive nanocarriers were simply prepared by the ringopening polymerization (ROP) of alpha-lipoic acid [27].

Herein, based on our previous work, we hypothesized that the combination of cytotoxic drugs and VDAs can achieve improved anti-tumor effect with selective release

\footnotetext{
${ }^{1}$ Key Laboratory of Polymer Ecomaterials, Changchun Institute of Applied Chemistry, Chinese Academy of Sciences, Changchun 130022, China

${ }^{2}$ University of Science and Technology of China, Hefei 230026, China

${ }^{3}$ Department of Chemistry, Northeast Normal University, Changchun 130021, China

* Corresponding authors (emails: ztang@ciac.ac.cn (Tang Z); xschen@ciac.ac.cn (Chen X))
} 
by GSH response in tumors. Honokiol (HNK), one ancient analogue of traditional Chinese medicine extracts, was chosen as a cytotoxic drug to form the conjugate with DMXAA (HNK-DMXAA) by chemical bonding. After the ROP of DL-alpha-lipoic acid (ALA) to obtain poly (alpha-lipoic acid) (PALA) [28,29] and subsequent PEGylation to obtain PEGylated poly(alpha-lipoic acid) copolymer (PALA- $g$-mPEG) in a simple way, the HNKDMXAA conjugate was grafted to PALA- $g$-mPEG to obtain the H-D NPs. When the H-D NPs arrived at the tumor tissues with high GSH concentration, HNK and DMXAA were released together due to the disintegration of the nanomedicine. Then DMXAA would block the blood supply and starve tumors to achieve internal necrosis, and HNK would kill the residual cells to achieve external death, which can realize the coordinated treatment of tumors, ultimately (Fig. 1).

\section{EXPERIMENTAL SECTION}

\section{Materials}

Poly(ethylene glycol) monomethyl ether $\left(M_{\mathrm{n}}=\right.$ $\left.5000 \mathrm{~g} \mathrm{~mol}^{-1}\right)\left(\mathrm{mPEG}_{5 \mathrm{k}}\right)$, ALA and reduced GSH were purchased from Sigma-Aldrich LLC (China). The 1-ethyl3-(3-dimethylaminopropyl)-carbodiimide hydrochloride
(EDC. $\mathrm{HCl}$ ) and $N, N^{\prime}$-diisopropylcarbodiimide (DIC) were purchased from J\&K Scientific Ltd. (China). The 4dimethylaminopyridine (DMAP) was purchased from Adamas Reagent, Ltd. (China). DMXAA was purchased from Changchun Third Party Pharmaceutical Technology Co., Ltd. (China). HNK was purchased from Dalian Meilun Biotechnology Co., Ltd. (China). Dulbecco's modified Eagle's medium (DMEM, Gibco) and fetal bovine serum (FBS, Gibco) were purchased from Thermo Fisher Scientific (China). Annexin V-FITC/PI apoptosis detecting kit was purchased from Nanjing KeyGen Biotech. Co. Ltd. (China). Terminal nucleotidyl transferasemediated nick end labeling (TUNEL) kit was purchased from F. Hoffmann-La Roche Ltd. (Switzerland). All the other reagents and solvents were purchased from Sinopharm Chemical Reagent Co., Ltd. (China).

\section{Characterization}

${ }^{1} \mathrm{H}$ NMR spectra were measured on a Bruker AV 300 NMR spectrometer in chloroform-d or dimethyl sulfoxide- $\mathrm{d}_{6}$. The zeta potential measurement was performed on a Zeta Potential/BI-90Plus particle size analyzer (Brookhaven Instruments Corporation, USA). Dynamic laser scattering (DLS) was tested by a WyattQELS instrument with a vertically polarized HeNe laser (Dawn

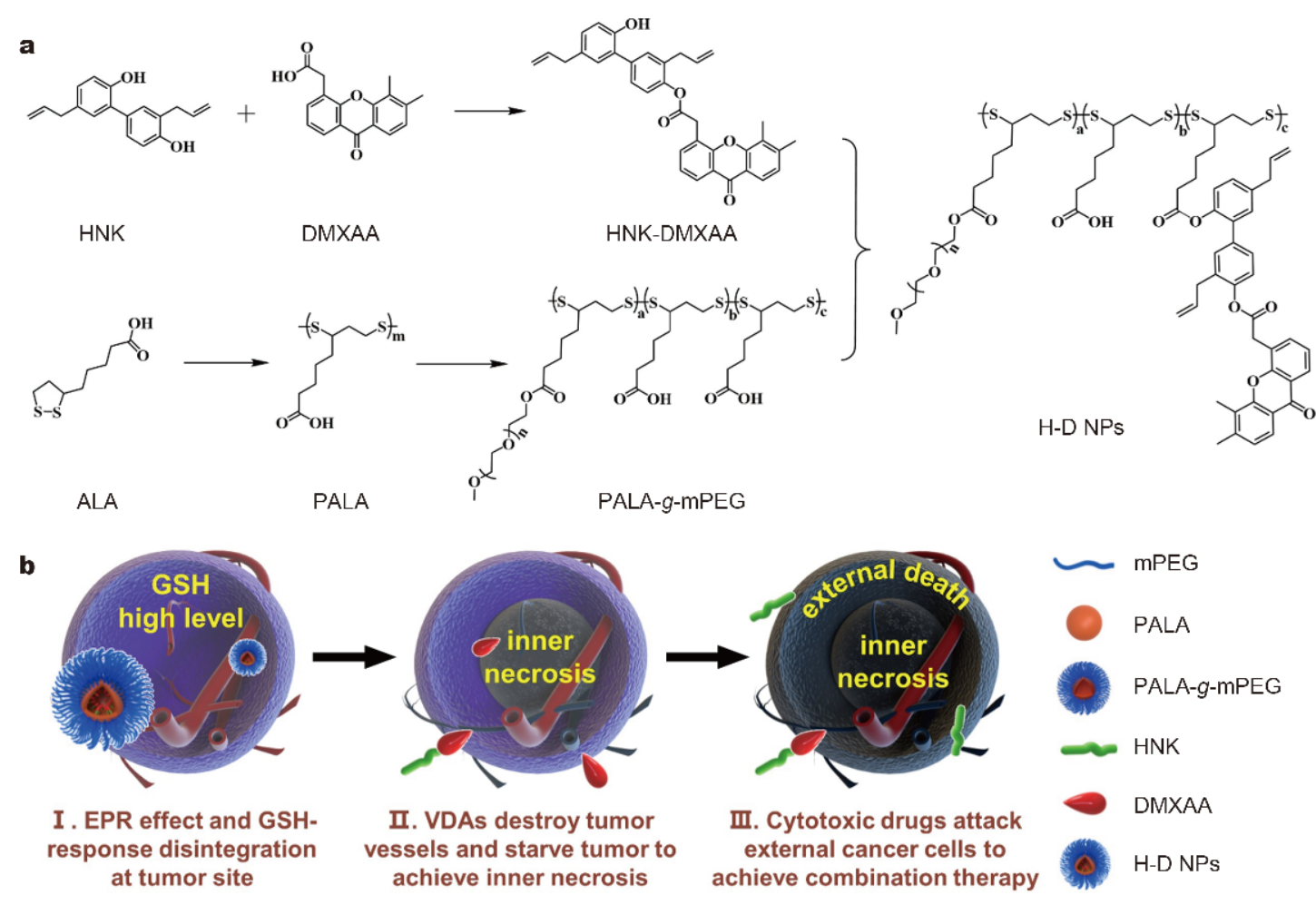

Figure 1 Synthesis (a) and mechanisms (b) of action of the H-D NPs. 
EOS, Wyatt Technology, USA). The scattering angle was fixed at $90^{\circ}$. The 3-(4,5-dimethyl-2-thiazolyl)-2,5-diphenyl-2- $H$-tetrazolium bromide (MTT) assay was tested on a microplate reader (INFINITE 200 PRO, USA). Electrospray ionization mass spectrometry (ESI-MS) was detected using a Thermo LTQ XL ion trap mass spectrometer (Thermo Finnigan, USA) in a negative ion mode $\left(\mathrm{ESI}^{-}\right)$. The fluorescence intensity was performed on fluorescence master system (Photon Technology International, USA). The high performance liquid chromatography (HPLC) analyses were measured on a Waters 1525 system equipped with a reverse-phase column (Symmetry®C18). Acetonitrile and water $(v / v=4: 1)$ were selected as the elution, pumped at a flow velocity of $1.0 \mathrm{~mL} \mathrm{~min}^{-1}$ at $25^{\circ} \mathrm{C}$.

\section{Cell lines and animals}

The human umbilical vein endothelial cell line (HUVEC) and the murine breast cancer $4 \mathrm{~T} 1$ cell line were purchased from Shanghai Bogoo Biotechnology Co. Ltd. (China). BALB/c mice (female, 6-8 weeks old, average body weight 18-20 g) were purchased from Beijing Vital River Laboratory Animal Technology Co. Ltd. (China). SD rats (male, 7-8 weeks old, average body weight 200-220 g) were purchased from Liaoning Changsheng biotechnology Co., Ltd. (China). The Balb/c mice breast tumor model was prepared by injecting $4 \mathrm{~T} 1$ cells $(2.0 \times$ $10^{6}$ per mouse) into subcutaneous of the right lower abdomen. All the animal experiments were conducted in accordance with the guidelines of the Laboratory Protocol of Animal Care and Use Committee, Jilin University.

\section{Synthesis of HNK-DMXAA}

The HNK-DMXAA conjugate was synthesized by Steglich esterification reaction. Specially, HNK (1.1 eq.), DMXAA (1.0 eq.), DIC (1.0 eq.) and DMAP (0.1 eq.) were dissolved into anhydrous dichloromethane (DCM), and stirred for $48 \mathrm{~h}$ in dark at room temperature. The product was isolated through silica gel column chromatography with eluent of DCM:petrol puriss $(\mathrm{PE})=1: 50$. The solvent was then removed by vacuum rotary evaporation.

\section{Synthesis of PALA- $g$-mPEG}

PALA- $g$-mPEG was synthesized as previously described with slight modification [27]. Briefly, ALA was added into a dry flask under a nitrogen atmosphere and stirred at $90^{\circ} \mathrm{C}$ for $1.5 \mathrm{~h}$. Then tetrahydrofuran (THF) was added to dissolve the crude product, and the product solution was slowly dripped into 10-fold volume of diethyl ether and precipitated for thrice. All the solvents were removed under vacuum to obtain dry PALA. Then, PALA (5.0 eq. on the base of ALA) was dissolved in THF. Meanwhile, $\mathrm{mPEG}_{5 \mathrm{k}}-\mathrm{OH}$ (1.0 eq.), EDC. $\mathrm{HCl}$ (1.1 eq.) and DMAP (0.2 eq.) were dissolved in dimethyl sulfoxide (DMSO), then mixed with PALA, and stirred for $36 \mathrm{~h}$ at $30^{\circ} \mathrm{C}$. The reaction mixture was dialyzed using a dialysis bag (MWCO $7.0 \mathrm{kDa}$ ) against deionized water for $24 \mathrm{~h}$, and PALA- $g$-mPEG was obtained after lyophilization.

\section{Synthesis of H-D NPs}

The HNK-DMXAA was grafted to the prepared PALA- $g$ mPEG by Steglich esterification. Briefly, PALA- $g$-mPEG, HNK-DMXAA and DIC were added in anhydrous $N, N$ dimethylformamide (DMF) and stirred for $48 \mathrm{~h}$ in dark at room temperature. The reaction mixture was slowly precipitated by 10 times volume of diethyl ether to obtain a beige-white product. After being resolved with DMF, the crude product was precipitated into diethyl ether again and the solvents were removed by vacuum filtration. Next, the crude product was dissolved in DMF and dialyzed against deionized water for $12 \mathrm{~h}$ in dark. Finally, the H-D NPs were obtained by freeze-drying. The drug loading content (DLC) and drug loading efficiency (DLE) of the H-D NPs were determined by fluorescence detector HPLC (FLD-HPLC) with two channels fluorescence detector at $220 \mathrm{~nm} E_{\mathrm{x}}$ and $380 \mathrm{~nm} E_{\mathrm{m}}$ for $\mathrm{HNK}$, and at $345 \mathrm{~nm} E_{\mathrm{x}}$ and $409 \mathrm{~nm} E_{\mathrm{m}}$ for DMXAA, respectively. The elution of acetonitrile and water $(v / v=4: 1)$ pumped at flow velocity of $1.0 \mathrm{~mL} \mathrm{~min}{ }^{-1}$ at $25^{\circ} \mathrm{C}$ :

DLC $\%=\frac{\text { weight of drug loaded in nanomedicines }}{\text { weight of drug-loaded nanomedicines }}$

$$
\times 100 \% \text {. }
$$

\section{Zeta potential and GSH-responsiveness}

PALA- $g$-mPEG $\quad\left(1.0 \mathrm{mg} \mathrm{mL}^{-1}\right)$ and H-D NPs $\left(1.0 \mathrm{mg} \mathrm{mL}^{-1}\right)$ were dissolved in deionized water, and then measured by the zeta potential analyzer. To measure the responsiveness of the nanomedicine, the H-D NPs $\left(2.0 \mathrm{mg} \mathrm{mL}^{-1}\right)$ were dissolved in phosphate buffered saline (PBS, pH 7.4, $100.0 \mathrm{mmol} \mathrm{L}^{-1}$ ) with or without $5.0 \mathrm{mmol} \mathrm{L}^{-1} \mathrm{GSH}$, shaken at $37^{\circ} \mathrm{C}$ for 0 or $24 \mathrm{~h}$, and then the sizes were measured by DLS. To study the GSH response of the nanomedicine more intuitively, further characterization was performed by transmission electron microscopy (TEM) as well.

\section{In vitro release}

In vitro releases of HNK and DMXAA from the H-D NPs were tested using a dialysis method. Briefly, PBS $\left(100.0 \mathrm{mmol} \mathrm{L}^{-1}\right)$ and PBS $\left(100.0 \mathrm{mmol} \mathrm{L}^{-1}\right)$ containing 
GSH $\left(0.1,1.0,5.0 \mathrm{mmol} \mathrm{L}^{-1}\right)$ were used as the release media. H-D NPs $(5.0 \mathrm{mg})$ were dissolved with $5.0 \mathrm{~mL}$ different release media. Next, the samples were transferred into the dialysis bags (MWCO $3.5 \mathrm{kDa}$ ), shaken in $45.0 \mathrm{~mL}$ release media with $85 \mathrm{rmin}^{-1}$ at $37^{\circ} \mathrm{C}$. At $0,2,4$, 8,12 and $24 \mathrm{~h}, 3.0 \mathrm{~mL}$ of outside solution was taken out and then $3.0 \mathrm{~mL}$ fresh media was added. The accumulative releases of HNK and DMXAA were monitored by FLD-HPLC with two channels fluorescence detector at $220 \mathrm{~nm} E_{\mathrm{x}}$ and $380 \mathrm{~nm} E_{\mathrm{m}}$ for HNK, or at $345 \mathrm{~nm} E_{\mathrm{x}}$ and $409 \mathrm{~nm} E_{\mathrm{m}}$ for DMXAA at real time, with the elution of acetonitrile and water $(v / v=4: 1)$ pumped at flow velocity of $1.0 \mathrm{~mL} \mathrm{~min}^{-1}$ at $25^{\circ} \mathrm{C}$, as described above.

\section{In vitro cytotoxicity and cell apoptosis analysis}

The cytotoxicity in vitro was tested by MTT assay. 4T1 or HUVEC cells were seeded in 96-well culture plates $(5.0 \times$ $10^{3}$ cells per well) with $160 \mu \mathrm{L}$ DMEM and $20 \mu \mathrm{L}$ FBS per well, then allowed to adhere for $12 \mathrm{~h}$. DMXAA or H-D NPs were dissolved in PBS to obtain $5.0 \mathrm{mmol} \mathrm{L}^{-1}$ stock solution based on DMXAA, respectively. HNK and HNKDMXAA conjugate were dissolved in DMSO, and then diluted with PBS to obtain $1.0 \mathrm{mmol} \mathrm{L}^{-1}$ stock solution, respectively. Next, $20 \mu \mathrm{L}$ of the solutions were added to each well and allowed to incubate for $24 \mathrm{~h}$. After that, $20 \mu \mathrm{L}$ of $5 \mathrm{mg} \mathrm{mL}^{-1}$ MTT in PBS was added to each well, and the cells were incubated for additional 3 min in dark at $37^{\circ} \mathrm{C}$. All the DMEM were emptied completely and $150 \mu \mathrm{L}$ DMSO was added to each well subsequently and shocked for $3 \mathrm{~min}$. The absorbance of the solution was measured on the microplate reader at $490 \mathrm{~nm}$. The relative cell viability was determined by comparing the absorbance of treated cells at $490 \mathrm{~nm}$ to non-treatment control wells. Data were presented as mean $\pm \mathrm{SD}(n=3)$. As for the cell apoptosis analysis, 4T1 cells were seeded in 6-well culture plates $\left(1.0 \times 10^{5}\right.$ per well $)$ with $1.8 \mathrm{~mL}$ DMEM and $0.2 \mathrm{~mL}$ FBS per well, then allowed to adhere for $12 \mathrm{~h} .100 \mu \mathrm{L}$ of PBS, HNK $\left(63 \mu \mathrm{mol} \mathrm{L}^{-1}\right)$, DMXAA $\left.(63 \mu \mathrm{mol} \mathrm{L})^{-1}\right)$ and H-D NPs $\left(63 \mu \mathrm{mol} \mathrm{L}^{-1}\right.$ on the basis of $\mathrm{HNK}$ ) were added to each well and allowed to incubate for $24 \mathrm{~h}$, respectively. After that, the apoptotic of $4 \mathrm{~T} 1 \mathrm{cells}$ was tested by Annexin V-FITC/PI apoptosis detecting kit and flow cytometry.

\section{Anti-tumor efficiency in vivo}

The Balb/c mice breast tumor model was constructed by subcutaneous injection of $4 \mathrm{~T} 1$ cells $\left(2.0 \times 10^{6}\right.$ per mouse $)$ into the right lower abdomen. The mice were divided into 5 groups ( 5 mice per group) randomly when the tumors grew up to about $120 \mathrm{~mm}^{3}$. Then they were injected with
PBS (100 mmol L ${ }^{-1}$, i.v.), HNK (10.0 $\mathrm{mg} \mathrm{kg}^{-1}$, i.p.), DMXAA (11.3 $\mathrm{mg} \mathrm{kg}^{-1}$, i.v.), small molecule combination of HNK (10.0 mg kg${ }^{-1}$, i.p.) and DMXAA $\left(11.3 \mathrm{mg} \mathrm{kg}^{-1}\right.$, i.v.), or H-D NPs (equal to $10.0 \mathrm{mg} \mathrm{kg}^{-1}$ of $\mathrm{HNK}$ and $11.3 \mathrm{mg} \mathrm{kg}^{-1}$ of DMXAA, i.v.) on $0,4,8,12$ and 16 day. The tumor size and body weight were measured every other day, and the tumor volume was calculated as $V=$ $a \times b^{2} / 2$ (a: the maximum diameter of tumors; $b$ : the minimum diameter of tumors).

\section{H \& E and immunohistochemical staining}

Two days after the last injection of anti-tumor efficiency test, the mice were sacrificed. Meanwhile, tumors were excised and fixed in 4\% PBS-buffered formaldehyde $(w / v)$ for $48 \mathrm{~h}$, embedded in paraffin and sliced at $5 \mu \mathrm{m}$, then stained with haematoxylin and eosin (H \& E) or CD31. As for CD31 staining, these fractions were in turn dewaxed and rehydrated with xylene, a series of graded ethanol, and deionized water. Endogenous peroxidase was quenched with $3 \%$ hydrogen peroxide for $15 \mathrm{~min}$. Next, the sliced samples were rinsed with deionized water, dipped in boiled citric buffer for $10 \mathrm{~min}$, blocked with blocking buffer (5\% normal goat serum) for $1.5 \mathrm{~h}$. And the slides were incubated with rabbit anti-CD31 (1:100) polyclonal antibody at $4^{\circ} \mathrm{C}$ overnight. After washing by PBS, goat anti-rabbit IgG (1:1000) was added and incubated for $1 \mathrm{~h}$ at $25^{\circ} \mathrm{C}$. The sections were stained by diaminobenzidine (DAB), counterstained with hematoxylin and mounted with mounting medium in proper sequence. The section stained brown showed CD31 positive.

\section{TUNEL assay}

TUNEL assay was measured according to the manufacturer's instructions on the TUNEL kit. And the apoptosis was measured by fluorescence microscopy.

\section{Statistical analysis}

Statistical analysis $(n=3)$ was expressed as the mean \pm SD standard deviation, using the One Way Anova. Statistically significant was expressed using ${ }^{\star}(p<0.05)$, highly significant was expressed using ${ }^{\star *}(p<0.01)$ and extremely significant was expressed using ${ }^{* *}(p<0.001)$.

\section{RESULTS AND DISCUSSION}

\section{Synthesis of HNK-DMXAA}

The HNK-DMXAA conjugate was characterized by ${ }^{1} \mathrm{H}$ NMR and ESI-MS in chloroform-d after isolated through silica gel chromatography column. As shown in Fig. S1, peak e of HNK-DMXAA shifted from $\delta 3.85 \mathrm{ppm}$ in 
DMXAA (Fig. S2) to $\delta 4.02 \mathrm{ppm}$, indicating that the HNK-DMXAA was successfully synthesized, and this was further confirmed by ESI-MS (Fig. S3), where the HNKDMXAA exhibited a peak at $m / z=529.6$ for $[\mathrm{M}-\mathrm{H}]^{-}$, while the $m / z=530.5$ was caused by isotopic molecules. This result indicated that the HNK-DMXAA conjugate was synthesized as expected.

\section{Synthesis of PALA- $g$-mPEG}

After ROP of alpha-lipoic acid monomer, PALA and PALA- $g$-mPEG were obtained and characterized by ${ }^{1} \mathrm{H}$ NMR in DMSO- $\mathrm{d}_{6}$. As shown in Fig. 2, as the dithiolane ring of ALA opened, peak a and c became overlapped, and peak 1 of mPEG appeared in PALA- $g$-mPEG after dialysis against deionized water for $24 \mathrm{~h}$, indicated that PALA was successfully synthesized and bonded to mPEG. The integral ratio of peak b to peak 1 in PALA- $g$-mPEG was 1:45, indicating that the mass ratio of PALA to $\mathrm{mPEG}$ was 5:1.

\section{Synthesis of H-D NPs}

The H-D NPs were characterized by ${ }^{1} \mathrm{H}$ NMR in DMSO$\mathrm{d}_{6}$. In Fig. 2, there appeared obvious benzene ring peaks from $\delta 7.20$ to $8.60 \mathrm{ppm}$, indicating that PALA- $g$-mPEG had successfully bonded to HNK-DMXAA. The DLC of HNK and DMXAA of H-D NPs was determined by FLDHPLC. As shown in Fig. 3, when fluorescence detector was set at $220 \mathrm{~nm} E_{\mathrm{x}}$ and $380 \mathrm{~nm} E_{\mathrm{m}}$, DMXAA only had a weak fluorescence signal at $4.60 \mathrm{~min}$, while HNK had a strong fluorescence signal at $7.06 \mathrm{~min}$. When fluorescence detector was set at $345 \mathrm{~nm} E_{\mathrm{x}}$ and $409 \mathrm{~nm} E_{\mathrm{m}}$, no fluorescence signal was found in HNK, while DMXAA had strong fluorescence signal in $4.56 \mathrm{~min}$. On this basis, we determined the DLC of HNK and DMXAA in the H-D NPs were $5.3 \mathrm{wt} \%$ and $4.7 \mathrm{wt} \%$, respectively. The slight difference in molar ratio (1.19:1) may be due to the partial cleavage of the conjugate of HNK and DMXAA during dialysis purification.

\section{Zeta potential}

The zeta potential of PALA- $g$-mPEG was $(-18.5 \pm$ 4.9) $\mathrm{mV}$, while the zeta potential of the H-D NPs changed to $(-12.8 \pm 5.8) \mathrm{mV}$, because the HNK-DMXAA bonding reduced the number of carboxyl group on PALA- $g$ mPEG. This further demonstrated that the H-D NPs were obtained successfully.

\section{GSH-responsiveness}

In order to verify the GSH responsiveness of the nanomedicine, H-D NPs were dissolved in PBS $\left(1.0 \mathrm{mg} \mathrm{mL}^{-1}\right)$ under different GSH concentrations. As shown in Fig. 4a,

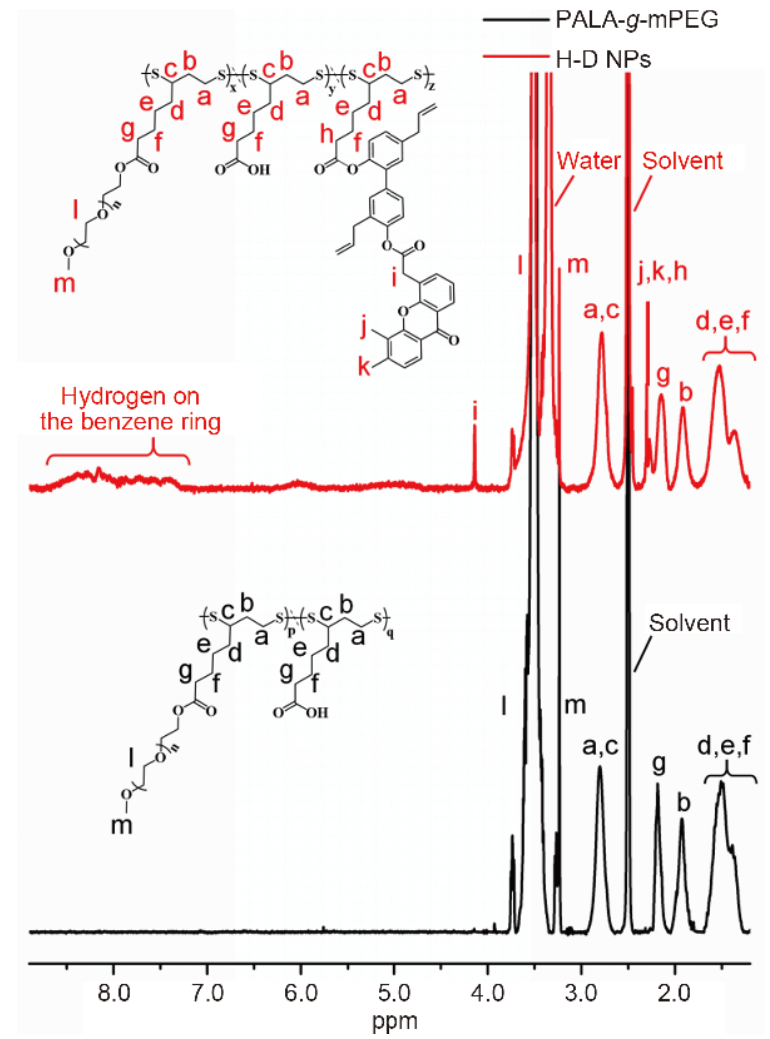

Figure $2{ }^{1} \mathrm{H}$ NMR spectra of PALA- $g$-mPEG and H-D NPs in DMSO- $\mathrm{d}_{6}$.
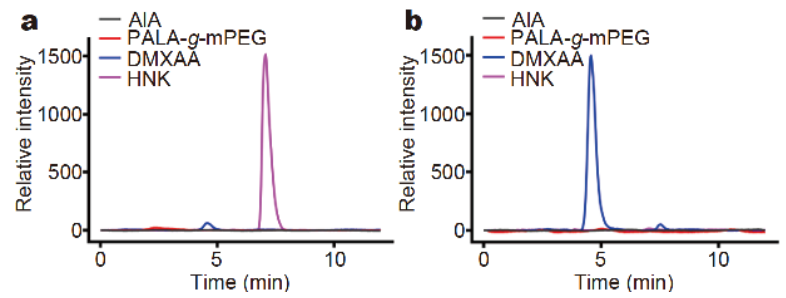

Figure 3 FLD-HPLC of ALA, PALA- $g$-mPEG, DMXAA and HNK with fluorescence detector at $220 \mathrm{~nm} E_{\mathrm{x}}$ and $380 \mathrm{~nm} E_{\mathrm{m}}$ (a) or $345 \mathrm{~nm} E_{\mathrm{x}}$ and $409 \mathrm{~nm} E_{\mathrm{m}}$ (b).

the hydrodynamic radius $\left(R_{\mathrm{h}}\right)$ of the H-D NPs in PBS was $(57.1 \pm 10.1) \mathrm{nm}$, and it became $(74.4 \pm 21.4) \mathrm{nm}$ after $24 \mathrm{~h}$, while the radius in PBS with $5.0 \mathrm{mmol} \mathrm{L}^{-1} \mathrm{GSH}$ changed to $(125.9 \pm 73.5) \mathrm{nm}$, which revealed the great GSH response ability of the nanomedicine. Meanwhile, to demonstrate the GSH response of the nanomedicine more intuitively, TEM measurement was carried out, which indicated that the average diameter of the H-D NPs in PBS (Fig. 4b) was about $(117.2 \pm 12.0) \mathrm{nm}$, while the disintegration of H-D NPs in Fig. 4c was obvious at the presence of $5.0 \mathrm{mmol} \mathrm{L}^{-1} \mathrm{GSH}$. The change of particle sizes also proved that the H-D NPs had excellent re- 


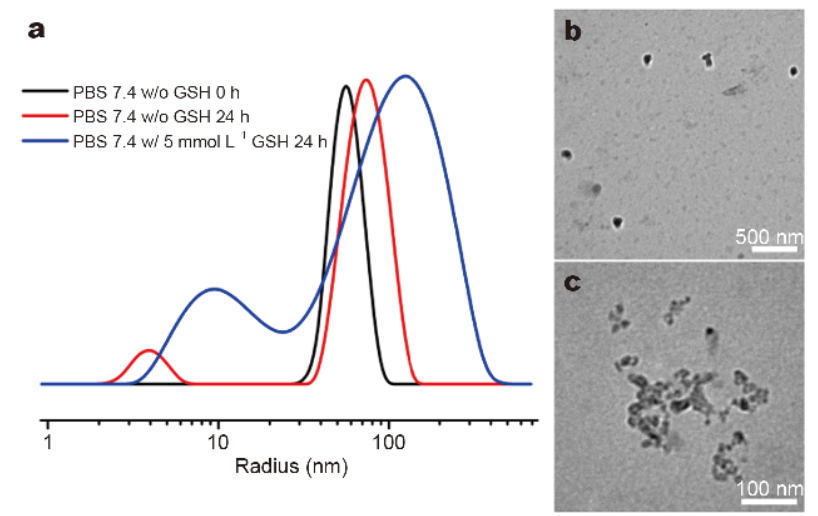

Figure 4 (a) Size changes of H-D NPs in different conditions (w/: with; w/o: without); TEM images of the H-D NPs in PBS without GSH (b) or with $5.0 \mathrm{mmol} \mathrm{L}^{-1} \mathrm{GSH}$ for $1 \mathrm{~h}$ (c).

sponsiveness to GSH.

\section{In vitro release}

To simulate the drug releases in the treatment, HNK and DMXAA releases in vitro at different GSH concentrations were measured. As shown in Fig. 5, the releases of both HNK and DMXAA increased with the disintegration of the H-D NPs by GSH. The HNK release in PBS with $5.0 \mathrm{mmol} \mathrm{L}^{-1} \mathrm{GSH}$ was 2.89-fold higher than that in PBS without GSH, while the DMXAA release in PBS with $5.0 \mathrm{mmol} \mathrm{L}^{-1} \mathrm{GSH}$ increased 2.75-fold higher than that in PBS without GSH. With the increase of GSH concentration, the drug release rate also increased, indicating the GSH responsive release of the nanomedicine.

\section{Cytotoxicity and cell apoptosis analysis}

To understand the cytotoxicity of drugs on cancer cells and vascular endothelial cells, 4T1 and HUVEC were used for MTT assay. As shown in Fig. 6, HNK, as a cytotoxic drug, had obvious cytotoxicity to both cell lines, with a half maximal inhibitory concentration $\left(\mathrm{IC}_{50}\right)$ of $11.5 \mu \mathrm{mol} \mathrm{L}^{-1}$ to $4 \mathrm{~T} 1$ and $\mathrm{IC}_{50}$ of $12.0 \mu \mathrm{mol} \mathrm{L}^{-1}$ to $\mathrm{HU}$ VEC. In contrast to HNK, DMXAA has inhibition at high concentrations on HUVEC cells, but no effect on 4T1 cells was exhibited. In addition, HNK-DMXAA exhibited similar cytotoxicity to $\mathrm{HNK}$, with an $\mathrm{IC}_{50}$ of $12.3 \mu \mathrm{mol} \mathrm{L}^{-1}$ to $4 \mathrm{~T} 1$ and $\mathrm{IC}_{50}$ of $10.88 \mu \mathrm{mol} \mathrm{L}^{-1}$ to HUVEC. Meanwhile, the H-D NPs exhibited lower inhibition on 4T1 and HUVEC due to the sustained-release property, with an $\mathrm{IC}_{50}$ of $142.5 \mu \mathrm{mol} \mathrm{L}^{-1}$ to $4 \mathrm{~T} 1$ and $\mathrm{IC}_{50}$ of $157.8 \mu \mathrm{mol} \mathrm{L}^{-1}$ to HUVEC, indicating that the HNK and DMXAA could be released from the H-D NPs. And as shown in Fig. S4, both HNK and H-D NPs can lead to the
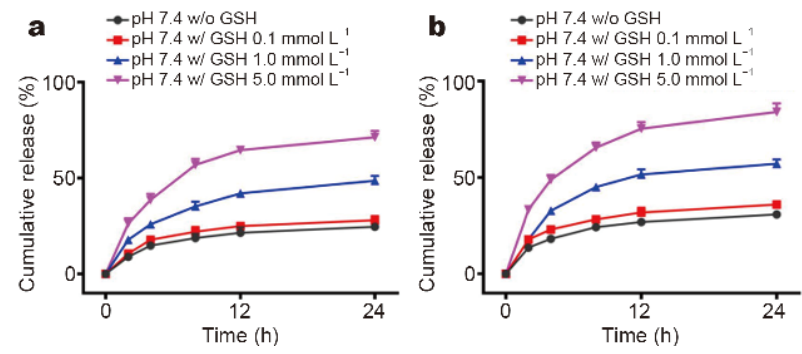

Figure 5 Accumulation releases of HNK (a) and DMXAA (b) from the H-D NPs (w/: with; w/o: without).
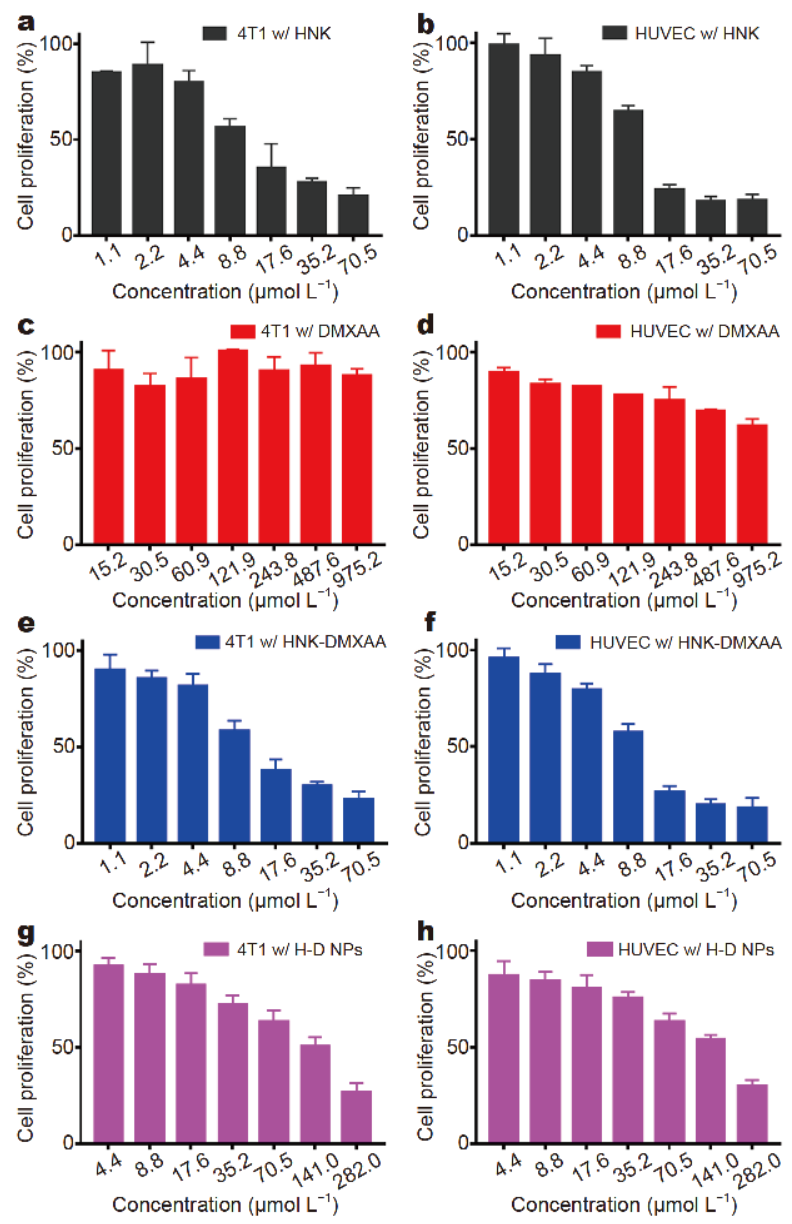

Figure 6 MTT assay of HNK (black), DMXAA (red), the HNKDMXAA conjugate (blue) and the H-D NPs (purple) to $4 \mathrm{~T} 1$ and HUVEC cell lines.

apoptosis of $4 \mathrm{~T} 1$ cells.

\section{Anti-tumor efficiency in vivo}

Owing to the excellent results in vitro, we were looking forward to the anti-tumor effect of H-D NPs in vivo. 4T1 model was chosen for anti-tumor efficacy research. As 
shown in Fig. 7, except PBS group, the tumor volumes all decreased, and only the group of small molecule drugs combination showed significant change in body weight during the treatment, and the inhibition rate of this group was $82.6 \%$, which was higher than $13.4 \%$ of $\mathrm{HNK}$ and $76.6 \%$ of DMXAA. As expected, H-D NPs had the strongest therapeutic effect and the inhibition rate was $93.0 \%$, which was significantly higher than other groups.

\section{H \& E staining}

To further study the effects of each drug on tumors, tumors after treatment were stained with $\mathrm{H} \& \mathrm{E}$ for pathological analysis. As shown in Fig. 8a-e, the tumor treated with PBS had large cell nuclei and abundant cytoplasm, indicating that the tumor had strong ability to proliferate. The tumor treated with HNK exhibited small cell nuclei and the morphology was abnormal, which revealed the cytotoxicity of HNK. In the DMXAA treatment group, the necrosis of the central part of the tumor was serious, indicating that the blocking effect of DMXAA on blood vessels blocked the supply of the tumors and led to large necrosis area of the central cells in the tumor. The combination of HNK and DMXAA small molecules demonstrated the death of both internal and external tumors. Best of all, the tumor treated with H-D NPs exhibited the largest area of cell death and abnormal cell morphology, which also made the group of H-D NPs have the best anti-tumor efficacy.

\section{Immunohistochemical staining and TUNEL assay}

In order to study the effect of treatment on tumor vessels, we performed a pathological analysis of CD31 staining. For CD31 staining, the blood vessels were thick and rich in PBS group (Fig. 8f). HNK group showed a slight inhibition of vascular growth (Fig. 8g), while the vascular inhibition was obvious in the DMXAA group (Fig. 8h), the combination group of small molecules (HNK and DMXAA) (Fig. 8i) and H-D NPs group (Fig. 8j). Among them, H-D NPs group had the lowest mean vessel density, which was 19-fold lower than that in PBS group (Fig. S5). To evaluate whether tumor growth inhibition was related to apoptosis, TUNEL staining was performed. As seen in Fig. S6, the tumors injected with H-D NPs exhibited high populations of apoptotic cells than PBS group.
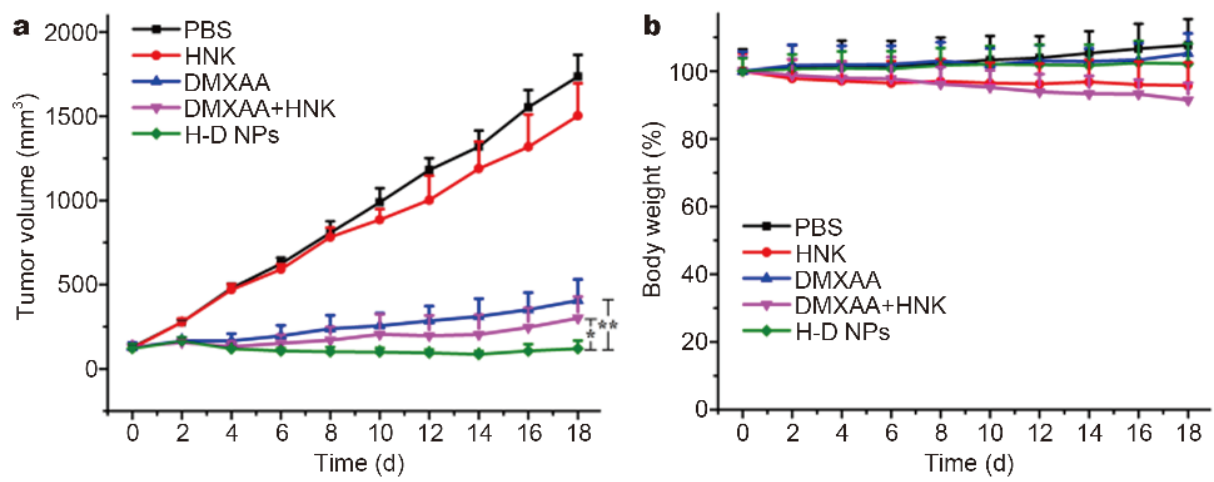

c

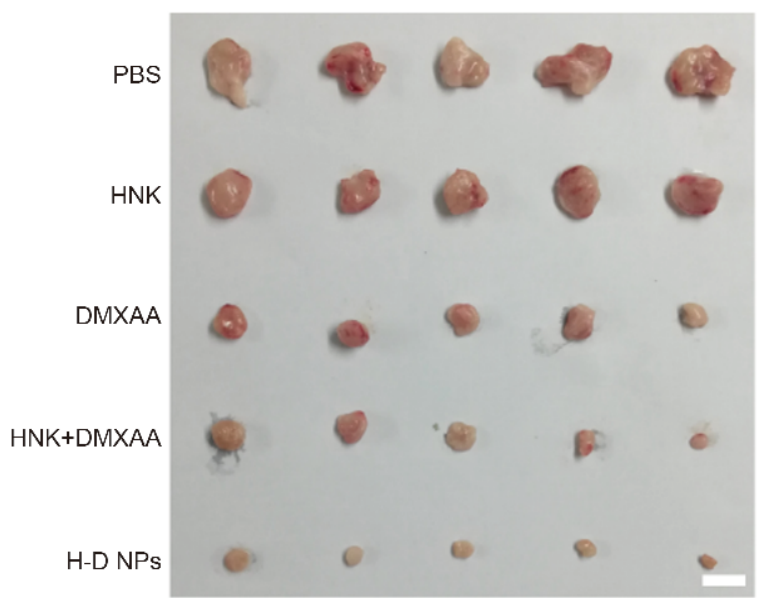

d

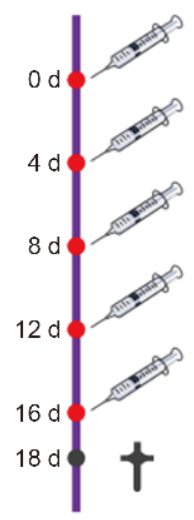

Figure 7 Anticancer efficiency and body weight changes in vivo. (a) Tumor volume and (b) body weight changes during therapy; (c) tumor images at the end of treatment; (d) injection schedule for all groups. Scale bar: $10 \mathrm{~mm}$. 


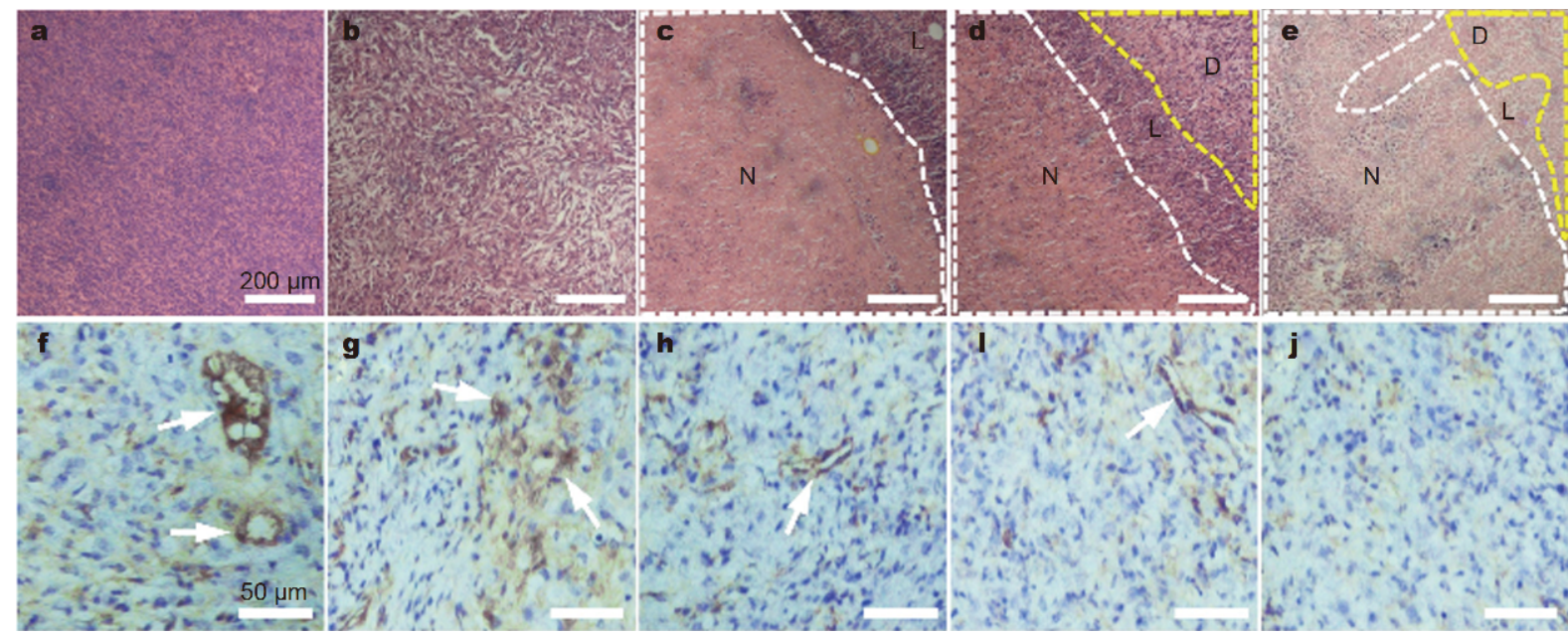

Figure 8 H \& E staining of PBS (a), HNK (b), DMXAA (c), HNK and DMXAA (d) and H-D NPs (E). N: necrosis; L: live; D: dead. Scale bar: $200 \mu$ m. And CD31 staining of PBS (F), HNK (G), DMXAA (H), HNK and DMXAA (I) and H-D NPs (J). White arrows show the location of blood vessels. Scale bar: $50 \mu \mathrm{m}$.

\section{CONCLUSIONS}

In summary, we developed a novel GSH-responsive nanomedicine, H-D NPs, by bonding the conjugate of HNK and DMXAA to PALA- $g$-mPEG. Due to the high level of GSH at the tumor sites, the H-D NPs could be disintegrated and achieve the co-delivery of HNK and DMXAA to the tumor sites. In addition, H-D NPs achieved combination therapy for $4 \mathrm{~T} 1$ breast cancer by blocking the blood supply of the inner tumor with DMXAA, killing the external cells of the tumor with HNK, which exhibited high tumor inhibition rate of $93 \%$. The approach reported here for cooperative therapy can be potentially applied towards other anti-tumor drugs. Furthermore, it will facilitate the progress in the field of combination therapy and help to advance clinical trials.

\section{Received 22 June 2019; accepted 10 September 2019;} published online 21 October 2019

1 Chen $\mathrm{H}, \mathrm{Gu} \mathrm{Z}$, An $\mathrm{H}$, et al. Precise nanomedicine for intelligent therapy of cancer. Sci China Chem, 2018, 61: 1503-1552

2 Chen J, Lin L, Yan N, et al. Macrophages loaded CpG and GNRPEI for combination of tumor photothermal therapy and immunotherapy. Sci China Mater, 2018, 61: 1484-1494

3 Hasani A, Leighl N. Classification and toxicities of vascular disrupting agents. Clin Lung Cancer, 2011, 12: 18-25

4 Daei Farshchi Adli A, Jahanban-Esfahlan R, Seidi K, et al. An overview on vadimezan (DMXAA): the vascular disrupting agent. Chem Biol Drug Des, 2018, 91: 996-1006

5 Bähr O, Gross S, Harter P, et al. ASA404, a vascular disrupting agent, as an experimental treatment approach for brain tumors. Oncol Lett, 2017, 14: 5443-5451

6 Hohenberger P, Elleni S, Marc F, et al. Vascular disrupting agent ASA 404 evaluated in human isolated ventilated and perfused lung lobes containing NSCLC. Eur J Cancer, 2015, 51: S608

7 Lv S, Tang Z, Song W, et al. Inhibiting solid tumor growth in vivo by non-tumor-penetrating nanomedicine. Small, 2017, 13: 1600954

8 Song W, Tang Z, Zhang D, et al. Solid tumor therapy using a cannon and pawn combination strategy. Theranostics, 2016, 6: 1023-1030

9 Cheng R, Meng F, Deng C, et al. Bioresponsive polymeric nanotherapeutics for targeted cancer chemotherapy. Nano Today, 2015, 10: 656-670

10 Cheng R, Meng F, Deng C, et al. Dual and multi-stimuli responsive polymeric nanoparticles for programmed site-specific drug delivery. Biomaterials, 2013, 34: 3647-3657

11 Ganta S, Devalapally H, Shahiwala A, et al. A review of stimuliresponsive nanocarriers for drug and gene delivery. J Control Release, 2008, 126: 187-204

12 Thornton P, Mart R, Ulijn R. Enzyme-responsive polymer hydrogel particles for controlled release. Adv Mater, 2007, 19: 12521256

13 Chen K, Liao S, Guo S, et al. Enzyme/pH-sensitive dendritic polymer-DOX conjugate for cancer treatment. Sci China Mater, 2018, 61: 1462-1474

14 Du JZ, Du XJ, Mao CQ, et al. Tailor-made dual pH-sensitive polymer-doxorubicin nanoparticles for efficient anticancer drug delivery. J Am Chem Soc, 2011, 133: 17560-17563

15 Du JZ, Sun TM, Song WJ, et al. A tumor-acidity-activated chargeconversional nanogel as an intelligent vehicle for promoted tumoral-cell uptake and drug delivery. Angew Chem Int Ed, 2010, 49: 3621-3626

$16 \mathrm{Xu} \mathrm{Q}, \mathrm{He} \mathrm{C}$, Xiao C, et al. Reactive oxygen species (ROS) responsive polymers for biomedical applications. Macromol Biosci, 2016, 16: 635-646

17 Yuan F, Li JL, Cheng $\mathrm{H}$, et al. A redox-responsive mesoporous silica based nanoplatform for in vitro tumor-specific fluorescence imaging and enhanced photodynamic therapy. Biomater Sci, 2017, 6: $96-100$

18 Chen D, Zhang G, Li R, et al. Biodegradable, hydrogen peroxide, and glutathione dual responsive nanoparticles for potential pro- 
grammable paclitaxel release. J Am Chem Soc, 2018, 140: 73737376

19 Elzes MR, Akeroyd N, Engbersen JFJ, et al. Disulfide-functional poly(amido amine)s with tunable degradability for gene delivery. J Control Release, 2016, 244: 357-365

20 Zhang P, Wu J, Xiao F, et al. Disulfide bond based polymeric drug carriers for cancer chemotherapy and relevant redox environments in mammals. Med Res Rev, 2018, 38: 1485-1510

21 Perry RR, Mazetta J, Levin M, et al. Glutathione levels and variability in breast tumors and normal tissue. Cancer, 1993, 72: 783787

22 Huang ZZ, Chen C, Zeng Z, et al. Mechanism and significance of increased glutathione level in human hepatocellular carcinoma and liver regeneration. Faseb J, 2001, 15: 19-21

23 Wolf CR, Lewis AD, Carmichael J, et al. The role of glutathione in determining the response of normal and tumour cells to anticancer drugs. Biochm Soc Trans, 1987, 15: 728-730

24 Schnelldorfer T, Gansauge S, Gansauge F, et al. Glutathione depletion causes cell growth inhibition and enhanced apoptosis in pancreatic cancer cells. Cancer, 2000, 89: 1440-1447

25 Lee MH, Yang Z, Lim CW, et al. Disulfide-cleavage-triggered chemosensors and their biological applications. Chem Rev, 2013, 113: 5071-5109

26 Nie JJ, Qiao B, Duan S, et al. Unlockable nanocomplexes with selfaccelerating nucleic acid release for effective staged gene therapy of cardiovascular diseases. Adv Mater, 2018, 30: 1801570

27 Liu Z, Shen N, Tang Z, et al. An eximious and affordable GSH stimulus-responsive poly( $\alpha$-lipoic acid) nanocarrier bonding combretastatin A4 for tumor therapy. Biomater Sci, 2019, 7: 28032811

28 Kisanuki A, Kimpara Y, Oikado Y, et al. Ring-opening polymerization of lipoic acid and characterization of the polymer. J Polym Sci A Polym Chem, 2010, 48: 5247-5253

29 Ishida H, Kisanuki A, Endo K. Ring-opening polymerization of aromatic 6-membered cyclic disulfide and characterization of the polymer. Polym J, 2009, 41: 110-117

Acknowledgements This work was supported by the Ministry of Science and Technology of China (2018ZX09711003-012), the National Natural Science Foundation of China (51873206, 51673189, 51829302, 51503202, 51833010 and 51520105004), and the Program of Scientific Development of Jilin Province (20190103033JH).

Author contributions Tang $\mathrm{Z}$ and Chen $\mathrm{X}$ put forward research ideas. Liu $\mathrm{Z}$ designed and synthesized the samples, $\mathrm{Si} \mathrm{X}$ and $\mathrm{Wu} \mathrm{J}$ performed some chemical synthesis experiments, Shen $\mathrm{N}$ and Zhang D performed the animal experiments; Liu $\mathrm{Z}$ wrote the paper with support from Tang $\mathrm{Z}$ and Chen $\mathrm{X}$. All authors contributed to the general discussion.

Conflict of interest The authors declare that they have no conflict of interest.

Supplementary information online version of the paper.

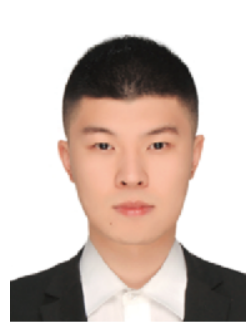

Zhilin Liu is a PhD candidate at the University of Science and Technology of China. His current research focuses on the tumor-microenvironment responsive nanomedicine of drug delivery system.

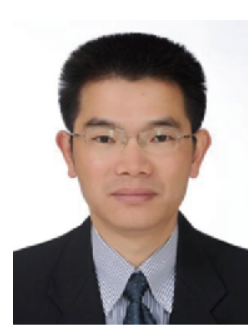

Zhaohui Tang is a professor in Changchun Institute of Applied Chemistry, Chinese Academy of Sciences. His research interests are focused on polymeric carriers for tumor treatment and combinational therapy. He has published more than 100 papers in SCI journals, such as Progress in Polymer Science, Advanced Materials, Advanced Science, Biomaterials, Small and Chemical Science.

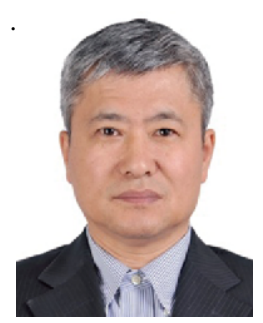

Xuesi Chen is a professor in Changchun Institute of Applied Chemistry, Chinese Academy of Sciences. His research interests are focused on polymers chemistry on biomedical polymers, biodegradable polymers for drug/gene controlled release, bone repair parts and tissue engineering scaffolds. He published more than 600 papers in SCI journals, such as Progress in Polymer Science, Advanced Materials, Journal of the American Chemical Society and Advanced Functional Materials. He was authorized more than 200 Chinese invention patents.

\section{一种新型GSH响应性聚硫辛酸纳米载体键合和厚 朴酚-DMXAA缀合物用于联合治疗}

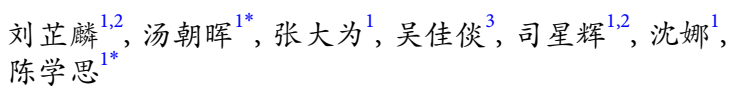

摘要 提高肿瘤治疗效果的关键在于增加药物肿瘤部位递送. 为 此, 肿瘤微环境刺激响应性材料具有很大的潜力. 本文通过将和厚 朴酚和DMXAA缀合物键合到GSH响应性聚硫辛酸-聚乙二醇纳米 载体上, 制备出一种新型纳米药物. 在肿瘤部位高谷胱甘肽水平时 纳米药物解体，实现和厚朴酚与DMXAA在肿瘤部位的共同递送. 通过和厚朴酚的近程攻击与DMXAA的远程杀伤, 协同治疗乳腺 癌. 在小鼠4 1 1乳腺肿瘤模型中, 该策略显示肿瘤抑制率高达 $93 \%$, 为肿瘤治疗提供了有价值的候选治疗方案. 